\title{
Grand Challenges in Nuclear Physics: A Long and Exciting Way to Go
}

\author{
Laura E. Marcucci ${ }^{1,2 *}$ \\ ${ }^{1}$ Department of Physics "E. Fermi," University of Pisa, Pisa, Italy, ${ }^{2}$ Sezione di Pisa, Istituto Nazionale di Fisica Nucleare, Pisa, \\ Italy
}

Keywords: hadron structure and dynamics, nuclear structure and dynamics, lepton-nucleus scattering, numerical methods for nuclear physics, nuclear reactions of astrophysical interest, neutron star

\section{INTRODUCTION}

Ordinary matter is made of atoms consisting of an electronic cloud surrounding a dense positively charged core. This core is known as the nucleus. Nowadays, after many years of intense research, we know that nuclei are made of positively charged protons and neutral neutrons, together called "nucleons." Nucleons themselves are also not structureless particles, but are made of quarks, bound together by the strong force, mediated by the gluons. Quarks and gluons are the degrees of freedom to be used when nuclei are investigated with high-energy probes (i.e., with energies higher than the nucleon mass itself). However, in ordinary matter, quarks are confined, and the relevant degrees of freedom are nucleons and eventually mesons. Nuclear Physics (NP) is that field of research dealing with nucleons inside nuclei.

The number of protons inside a nucleus corresponds, in neutral atoms, to the number of electrons surrounding the nucleus. Therefore, the number of protons determines the chemical characteristics and physical properties of a given element, as classified on the Periodic Table. Each element, though, can also have a different number of neutrons inside its nucleus. Elements having the same number of protons, but different number of neutrons are called isotopes. There are more than 250 isotopes on Earth, and more than thousands have been synthesized in laboratories, although most of them are unstable. The nuclear chart (see Figure 1), where these isotopes are classified according with the number of protons and neutrons, is definitely much more complex than the more familiar Periodic Table! It is then clear why NP is still nowadays a fascinating field of research, with many open questions, whose answers will advance our basic knowledge of Nature. Some of these questions are listed in section 2, the grand challenges in NP for the next 20 years or so.

The interest in NP is not limited to this. Many grand challenges in other fields, like High Energy Particle and Astroparticle Physics, Astronomy and Astrophysics, Cosmology, require to have under control several NP issues. In a sense, NP can be seen as at service for other fields. Also in this case, some grand challenges can be identified and are listed in section 3.

Needless to say, the list of items presented below is not exhaustive, and I apologize for its incompleteness. However, the list should provide a flavor of the breath of NP research.

\section{BASIC KNOWLEDGE OF NATURE}

Received: 13 June 2018 Accepted: 02 July 2018 Published: 20 July 2018

Citation:

The ultimate goal of NP is to unravel the fundamental properties of nuclei from their "building bricks," the nucleons, and ultimately to find the connection with the realm of Quantum Chromodynamics (QCD). In other words, NP should be able to start from an understanding of the internal structure of nucleons (and hadrons, more in general), and consequently of their mutual interaction, in order to describe the whole nuclear chart, from the few-nucleon systems, to heavier and heavier nuclei, to ultimately nuclear and neutron matter. This is of course a very ambitious 


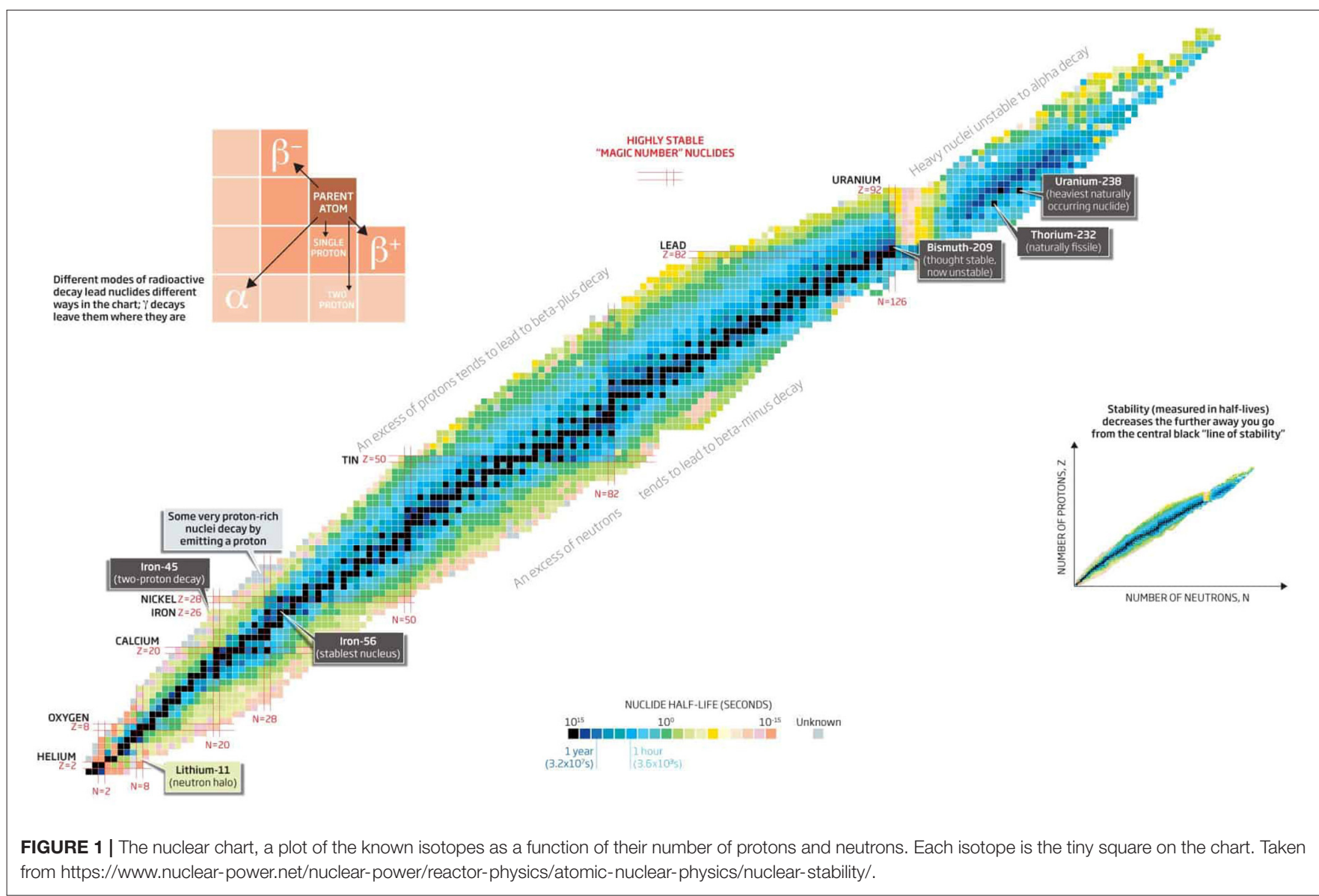

program, a "grand grand challenge," for which we can identify some essential items. I list them, schematically, in the following.

- A grand challenge for NP is to have a detailed knowledge of the structure of hadrons, possibly getting informations on key aspects, such as the spatial distribution of quarks in the proton, or the connection between the quark spin and orbital angular momentum and the spin of the proton. The three-dimensional description of the nucleon, achievable through the socalled Transverse Momentum-Dependent parton distribution functions (TMDs), is also a grand challenge, both theoretically and experimentally. Relative to the proton structure, the inconsistency between electron-scattering and the muonic hydrogen results for the proton radius, the so-called "proton radius puzzle," needs to be solved (for a recent review see [1]).

- Nuclear force between nucleons can be seen as a residual force resulting from the constituents of the nucleons, i.e., the quarks. How this force is connected with QCD has been an intense field of research since many decades. Thanks to lattice QCD and to the advent of chiral effective field theory (for recent reviews see $[2,3]$ ), we are in the process of building nuclear interaction on the strong basis of QCD. Some issues however remain unsolved and definitely constitute grand challenges for NP: while the nucleon-nucleon interaction is nowadays quite well under control, the same cannot be said for the three-nucleon force, for which at the moment exist far less sophisticated models. This reflect on some longstanding discrepancies between theory and experiment in fewbody observables, as the well known " $A_{y}$ puzzle" (see [4] and references therein). The solution of this puzzle is a grand challenge for NP. The situation is even worse for the hyperonnucleon and hyperon-hyperon interactions, for which lot of work still needs to be done. This is ultimately related to the "hyperon-puzzle" in neutron stars [5], which will be discussed below.

- Nuclei are often involved in nuclear reactions mediated by the electromagnetic or weak interaction, as well as in lepton-nucleus scattering processes. It is then unavoidable to give an appropriate description of the interaction of nuclei with electroweak external probes. A grand challenge for NP is the construction of nuclear electroweak currents within the same chiral effective field theory framework used for the nuclear interaction (for a recent review on nuclear electromagnetic currents and the electromagnetic structure of light nuclei see [6]). Ultimately, a consistent treatment of nuclear reaction mechanisms for heavier and heavier systems is highly desirable, in order to build a bridge between theory and experiment. 
- In order to assess the above mentioned items, as well as the ones which will be mentioned below, it is mandatory to develop robust and accurate numerical methods to solve the quantum mechanical problem, from the few- to the manybody realm, and even to infinite matter. A large effort has been already done so far, with very accurate techniques existing especially for light and intermediate-mass nuclei. But even here, where the calculation should be easier, the available methods struggle in the case of scattering states. But scattering states are essential in dealing with nuclear reaction. A grand challenge for NP, therefore, will be to develop highly accurate numerical techniques both for bound and scattering states, in order to solve more and more complex systems. It is clear that none of them will work for the whole mass range, but it will be crucial to find overlaps in the nuclear chart, which are the arrival point for the techniques working up to that level, and the starting point for those working from that level on. In this context, benchmark calculations will become essential.

\section{NUCLEAR PHYSICS AT SERVICE FOR OTHER FIELDS}

NP is a research field with lot of connections with other fields. Therefore, the grand challenges for them reflect on grand challenges for NP. We list below what we believe are the most relevant ones.

- Neutrino physics: Neutrinos remain among the most intriguing particles of the Standard Model. They are not massless, but their mass is extremely small, they are the only particles which interact only through the weak force, making their detection a great challenge for experimentalists. In fact, we even do not know the ultimate nature of neutrinos. It is natural that they are the object of intense research. Many accelerator experiments are running, or planned to run, with the ultimate goal of measuring neutrino-oscillation parameters, as the neutrino mass hierarchy and the chargeconjugation parity violating phase. These experiments employ nuclear targets, like ${ }^{16} \mathrm{O},{ }^{56} \mathrm{Fe},{ }^{208} \mathrm{~Pb}$, or ${ }^{40} \mathrm{Ar}$, and simple models for the nucleus and reaction mechanism. A grand challenge of NP is to provide an accurate determination of the neutrino-nucleus cross section in a wide energy range, to become accurate inputs for the analysis of experimental data.

The detection of neutrino-less double beta decay is nowadays one of the main targets in many laboratories all around the world, in order to reveal whether neutrinos are Dirac or Majorana particles. The observation of such a process would be the evidence of lepton number violation and a clear evidence of "new physics" beyond the Standard Model. The decay rate is proportional to a nuclear matrix element, which involves the initial and final nuclei wave functions. The accurate calculation of this matrix element, also in order to identify which nuclear systems is more convenient for experiment, is mandatory, and indeed it represents again a grand challenge for NP.
- Astrophysics, from cosmology and the theory of Big Bang Nucleosynthesis (BBN) to stellar and solar physics: There are several astrophysical environments where nuclear reactions take place and are responsible ultimately for the picture that we have nowadays of the Universe. Soon after Big Bang, primordial light nuclei were formed during $\mathrm{BBN}$. In order to predict their abundances, the cross section of the relevant nuclear reactions and cosmological and standard physics inputs are necessary. The comparison of $\mathrm{BBN}$ predictions with astronomical observations will result on a test of both cosmological and fundamental physics models. Therefore, the knowledge of the nuclear cross sections, with the smallest possible uncertainty, is of paramount importance in this framework, and it is a grand challenge, both for theorists and experimentalists. At the end, any persisting discrepancy between predicted and observed primordial abundances would open up for the possibility of non-standard physics.

The study of the nuclear reactions involved in stellar evolution, both in non-explosive and explosive environments, is also fundamental to understand how stars "live" and eventually "die." The grand challenges mentioned in section 2 regarding nuclear structure and nuclear reactions find their application also in this field.

- Physics beyond the Standard Model: A large research activity all around the world is devoted to the search of the so-called dark matter. Experiments, typically performed underground, use detector filled with liquid argon or xenon. In order to understand the eventual signal from dark matter candidates, it is necessary to have the "standard" physics well under control. Only after this will be done, a discovery can be claimed. The description of the nuclear structure of the involved nuclei, as well as the mechanisms of the nuclear reactions between the detector nuclei and "standard" particles obviously represents a grand challenge for NP.

NP can play a major role also in studying the laws which govern physics at the most fundamental level. The discovery of parity violation in weak interaction is a notable example. Intense research on other symmetry violations is ongoing, both experimentally and theoretically, as in the case of time-reversal violating observables, like the nuclear electric dipole moments [7]. The measurement of such observables, to be compared with accurate theoretical "standard" and "non-standard" predictions, represents a grand challenge for NP.

- Multi-messenger physics: Crucial questions for the understanding of our Universe is: what is the nature of matter under extreme conditions? Can multi-messenger observations provide access to conditions not reached at present laboratories? Ultimately, what is the physics of neutron stars and binary mergers? These questions have become of paramount importance after the detection of the first gravitational wave, coming from the merger of two neutron stars, by the LIGO and Virgo detectors on 17 August 2017 (GW170817), followed by the aftermath of this merger seen in the electromagnetic spectrum. In order to understand neutron stars, it is important to constrain the equation of state (EoS) of neutron-rich strongly 
interacting matter [8]. Although the EoS of symmetric nuclear matter has been constrained over a wide range of densities around the saturation density, the spread of values at high densities, clearly of interest for neutron stars, still remains large. Also the knowledge of asymmetric nuclear matter is very limited, mainly because of difficulties in determining accurately the symmetry energy. All these issues represent grand challenges in NP. Last, but not least, a long-lasting puzzle which needs to be solved in the next years is the so-called "hyperon-puzzle" [5]. The composition of matter at the supra-saturation densities reached in neutron star cores is very uncertain. Particles other than nucleons and electrons are expected to appear, among

\section{REFERENCES}

1. Carlson CE. The proton radius puzzle. Progr Part Nucl Phys. (2015) 82:59-77. doi: 10.1016/j.ppnp.2015.01.002

2. Machleidt R. Historical perspective and future prospects for nuclear interactions. Int J Modern Phys E (2017) 26:1730005. doi: 10.1142/ S0218301317300053

3. Machleidt R, Sammarruca F. Chiral eft based nuclear forces: achievements and challenges. Phys Scrip. (2016) 91:083007. doi: 10.1088/0031-8949/91/8/083007

4. Tornow W, Esterline JH, Weisel GJ. Energy dependence of the threenucleon analyzing power puzzle. J Phys G Nucl Part Phys. (2008) 35:125104. doi: 10.1088/0954-3899/35/12/125104

5. Bombaci I. The hyperon puzzle in neutron stars. JPS Conf Proc. (2017) 17:101002. doi: 10.7566/JPSCP.17.101002

6. Marcucci LE, Gross F, Penã MT, Piarulli M, Schiavilla R, Sick I, et al. (2016). Electromagnetic structure of few-nucleon ground states. J Phys G Nucl Part Phys. 43:023002. doi: 10.1088/0954-3899/43/2/023002 which hyperons. The appearance of additional degrees of freedom tends to soften the EoS and lower the maximum possible mass of the neutron star. This is difficult to reconcile with a measured two solar mass neutron star. However, the available EoS are based on poorly known hyperonic two- and three-body interaction models, as mentioned before. A grand challenge of NP is the solution of this puzzle.

\section{AUTHOR CONTRIBUTIONS}

The author confirms being the sole contributor of this work and approved it for publication.

7. Engel J, Ramsey-Musolf MJ, van Kolck U. Electric dipole moments of nucleons, nuclei, and atoms: the standard model and beyond. Prog Part Nucl Phys. (2013) 71:21-74. doi: 10.1016/j.ppnp.2013.03.003

8. Oertel M, Hempel M, Klah̆n T, Typel S. Equations of state for supernovae and compact stars. Rev Mod Phys. (2017) 89:015007. doi: 10.1103/RevModPhys.89.015007

Conflict of Interest Statement: The author declares that the research was conducted in the absence of any commercial or financial relationships that could be construed as a potential conflict of interest.

Copyright (c) 2018 Marcucci. This is an open-access article distributed under the terms of the Creative Commons Attribution License (CC BY). The use, distribution or reproduction in other forums is permitted, provided the original author(s) and the copyright owner(s) are credited and that the original publication in this journal is cited, in accordance with accepted academic practice. No use, distribution or reproduction is permitted which does not comply with these terms. 